\title{
Quaderni
}

QUADERNI Communication, technologies, pouvoir

68 | Hiver 2008-2009

Militantisme médical et fabrique des politiques de santé

\section{Ce que revendiquer veut dire. Éléments pour une pragmatique de la monstration}

Avant-propos

\section{Virginie Tournay}

\section{(QpenEdition}

\section{Journals}

Édition électronique

URL : http://journals.openedition.org/quaderni/172

DOI : $10.4000 /$ quaderni. 172

ISSN : 2105-2956

\section{Éditeur}

Les éditions de la Maison des sciences de l'Homme

Édition imprimée

Date de publication : 5 janvier 2009

Pagination : 5-9

\section{Référence électronique}

Virginie Tournay, «Ce que revendiquer veut dire. Éléments pour une pragmatique de la monstration », Quaderni [En ligne], 68 | Hiver 2008-2009, mis en ligne le 05 janvier 2012, consulté le 24 septembre 2020. URL : http://journals.openedition.org/quaderni/172 ; DOI : https://doi.org/10.4000/quaderni.172 


\section{$D$ ossier}

\section{avant-propos : \\ Ce que}

revendiquer

veut dire.

Éléments

pour une

pragmatique

de la

monstration $^{1}$

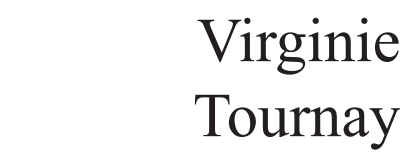

CNRS, IEP-PACTE / Grenoble
Le sage, a dit un ancien, est à la femme ce que l'asymptote est à la courbe ; il s'en approche toujours et n'y touche jamais.

C'est un demi-fou, nommé Rousseau, qui a énoncé cette belle maxime.

Gabriel Tarde, Fragments d'Histoire Future

Au premier regard, il ne semble guère approprié d'amorcer un avant-propos relatif aux activités revendicatives par une citation ironique de Gabriel Tarde sur l'agir moral et la maîtrise du désir. Pourtant, la lecture de l'incipit invite à qualifier les manières humaines d'intervenir dans un espace donné ou plus exactement, à discuter de la délicate confrontation entre un monde commun partagé par tout sujet percevant et le regard conscientisé pourvu des schèmes de référence, des valeurs ainsi que des interdits culturels. À l'évidence, les manières de s'engager visuellement dans le monde ne sont pas univoques mais composites, elles ne sont ni données, ni acquises une fois pour toutes car elles supposent la poursuite active d'un projet, d'une enquête de sens commun pour s'inscrire dans le monde extérieur, le qualifier et le confronter. Que l'on regarde un paysage, un défilé de mode, un corps dénudé, un drapeau flottant librement dans les airs ou une publicité célébrant les mérites d'une nouvelle médication, l'expérience visuelle est avant tout et immédiatement une qualification de l'objet qui s'y trouve visé. Cette visée est tantôt une appréhension, un engagement esthétique ou civique, tantôt une expérience ordinaire, un désir, le constat d'une performance, l'ajustement à une histoire spécifique ou l'expression d'un positionnement politique. Toute posture visuelle est derechef une manière située de relier au monde extérieur [Mills, 1940], elle est ainsi éminemment 
symbolique [Sfez, 1999] $]^{2}$ et constitutivement incertaine dans son accomplissement [Goffman, 1987 ; Dewey, 1967]. Si les manières de nous connecter visuellement à l'environnement renvoient à un processus immédiat, complexe et enchevêtré, cela ne signifie pas pour autant que la conduite et l'orientation de notre engagement au monde extérieur soient arbitraires. Il ne faut pas y préjuger la manifestation du libre arbitre d'une pure subjectivité ; le rapport visuel renvoie plutôt à un engagement situé car sa construction, quoique immédiate, est incontestablement contrainte et la pertinence de cet engagement est imposée par le contexte comme en attestent les travaux de sociologie phénoménologique [Schütz, 1970]. La perception est donc un processus actif de saisissement, où l'individu se positionne vis-àvis de quelque chose de préalable avec lequel il entre en rapport ; les alternatives contestataires ou l'ignorance des informations extérieures sont encore des formes de saisissement, et donc d'engagement à l'égard du monde extérieur [Trom, 1999].

Cet angle d'attaque phénoménologique inscrit un double présupposé. Tout d'abord, l'acte de revendiquer forme un cas spécifique d'attachement du sujet au monde extérieur, indissociable des systèmes de contraintes matérielles, des outillages opérationnels et des caractéristiques de figuration de l'objet qui est visé [Tournay, 2007a et b]. Penser les fondations de l'activité revendicative s'avère indissociable des conditions amenant à l'efficacité de la monstration. Cette mise à mal du dogme de l'immaculée perception [Frideman, 1967] nous oblige alors à braquer le projecteur sur l'efficacité pratique de l'acte de montrer, c'est-à-dire à penser une pragmatique de la monstration. Il s'agit de réfléchir aux outillages et aux procédés de la monstration, à leurs aptitudes à organiser un théâtre de la preuve, à relier au monde extérieur dans une direction voulue, à faire percevoir immédiatement et ainsi même, à provoquer l'adhésion. Plutôt que la démonstration qui implique une explicitation des règles du jeu symbolique et un effort de compréhension, l'activité revendicative s'appuie sur la monstration, sur la capacité à enrôler, à persuader et à séduire par le pouvoir d'immédiateté de la monstration. Si l'autorité sociale des porte-parole joue un rôle certain dans l'efficacité des propos, l'indexation hybride des productions discursives à des schèmes communs et à des registres de neutralité scientifique semblent renforcer leur pouvoir de conviction [Siméant, 2002]. En arrière-plan des travaux présentés dans ce numéro, la question de la persuasion et, plus particulièrement, la double problématique de la puissance militante des discours savants et de l'objectivation scientifique des énoncés vindicatifs se situe au cœur de ce qui définit l'envergure et l'efficacité de l'action collective. On ne saurait donc ici sous-estimer l'importance des particularités endogènes à l'énonciation dans l'efficacité de la monstration comme le mentionnent certaines des contributions à ce numéro. Le deuxième présupposé est la conséquence directe de notre constat de dénaturalisation du rapport visuel au monde. On peut affirmer ici que les espaces de monstration sont infinis. Dominés par un principe d'exposition, les lieux de l'art tels que les musées, les galeries, les festivals ou les lieux scientifiques et techniques, les laboratoires et les conférences professionnelles de recherche/développement forment bien évidemment, des espaces privilégiés de la monstration. Mais comme les faits divers 
de nos journaux en attestent régulièrement, tout évènement du quotidien confrontant l'expérience ordinaire de chacun est susceptible d'induire l'assentiment, l'approbation ou la colère. Ainsi et plus généralement, toute préhension visuelle invite à la mise en rapport d'éléments éparses et à une opération de figuration et d'assemblage en un tout cohérent. Les espaces potentiels de monstration sont donc omniprésents autour de nous. Et davantage encore, les instruments de monstration sont traduits en permanence dans des arènes diversifiées. Ainsi, Janelle Taylor [2008] montre comment les technologies à base d'ultrasons ont progressivement envahi la société de consommation. En travaillant le mode d'existence publique de cet instrument de monstration, c'est également la diversité des manières de faire preuve dans l'espace public, de désigner la réalité fœtale à partir d'un même outil opérationnel que l'auteur révèle.

Ce numéro de Quaderni propose de regarder de plus près les opportunités de monstration, et de nous intéresser plus particulièrement à celles qui sont symptomatiques des activités revendicatives, puis de suivre les modalités par lesquelles des pratiques ou des groupes controversés accèdent progressivement et aux yeux de tous, à la dignité de catégories stables et consolidées, notamment au sein de pratiques administratives et disciplinaires. On le voit à travers les différentes contributions, il est impossible de décoder les mécanismes de persuasion sans pénétrer les univers qui déploient plus ou moins efficacement les activités de monstration, voire de s'intéresser comme l'évoque Francis Sanseigne, aussi bien aux promotions de causes collectives ayant réussi qu'à celles qui ont échoué. En cela, les registres de protestations engagés dans le long cheminement vers l'objectivation [Tournay, 2007b] constituent une catégorie particulière de monstration. Elle se caractérise par une singulière puissance d'expression, marquée par des sauts d'espaces publics ainsi que par une extension des revendications particularistes à un souci du bien collectif. Des revendications telles que certaines formes de militantisme thérapeutique (Carlos Novas) ou de fin de vie (Michel Castra), trouvent ainsi une stabilisation dans le droit et sous la forme d'opérateurs de politique publique, elles incarnent alors une préoccupation d'intérêt général et une pragmatique de la démocratie [Giraud et Warin, 2008]. Qu'il s'agisse de déployer un activisme statistique (Sandrine Musso), d'introduire une expertise qui promeut un nouveau segment médical (Michel Castra), de bâtir un scandale (Francis Sanseigne) ou des récits de vie bénéficiant d'une large audience, ces processus de monstration nous renseignent, en dépit de leurs particularités contextuelles, sur les conditions d'entretien et d'institutionnalisation d'une cause collective. Carlos Novas met en évidence un mécanisme original d'entretien d'une mobilisation médicale : il montre l'importance des recadrages successifs des revendications de groupes de patients au sujet de la légalisation des traitements pour les maladies rares, le glissement progressif vers des débats sur les conditions d'accès à ces thérapeutiques. On voit alors poindre les revendications d'une citoyenneté thérapeutiques [Nguyen, 2005]. Bien que traduite et stabilisée dans le droit, une protestation collective institutionnalisée peut donc également déployer des postures de l'engagement visant à capter l'attention publique par de nouvelles actions [Fillieule, $1993]^{3}$. Les analyses présentées dans ce numéro de Quaderni déclinent les conditions de l'échec, 
de la réussite et de la transformation du processus d'objectivation des revendications sociales, que ces dernières passent par des associations de malades, une catégorie de professionnels de santé ou des acteurs de la société civile.

C'est alors autant une sociologie de la fondation que de l'action qui est ici travaillée. Et de la même manière que s'interrogent Stéphane Cadiou et Stéphanie Dechezelles concernant les mobilisations émergentes [2007], il ne s'agit pas tant de répondre à la question « pourquoi les hommes se révoltent-ils? » que de se demander « pourquoi ne le font-ils pas plus souvent ? »(p. 13). Cela implique de retracer les instruments déployés dans les mouvements émergents, d'identifier ceux qui détiennent une efficacité de monstration, c'està-dire de suivre ceux qui sont progressivement incorporés dans les pratiques disciplinaires, voire dans l'action publique [Lascoumes et Le Galès 2004] et de comprendre pourquoi d'autres n'y parviennent pas. La multiplicité des chemins pour parvenir à l'objectivité suppose des outils opérationnels qui prennent en charge la médiation entre celui qui perçoit et l'objet perçu de revendications. Ces outils de monstration peuvent être des narrations et coïncider avec les schèmes culturels d'un groupe donné [Dodier et Barbot, 2008] ${ }^{4}$ ou renvoyer à des supports technologiques comme le microscope par exemple ou plus généralement, correspondre à tout dispositif articulant l'observateur à un réel non directement perceptible [Dumit, 2003 ; Prentice, 2005] $]^{5}$. Suivant une succession d'épreuves indissociablement matérielles et sociales, ces instruments de l'action collective vont rapporter la preuve de la consistance de catégories ou de pratiques nouvelles. On en déduit le constat suivant : cette approche suppose de considérer le discours militant, non pas comme un énoncé intrinsèquement subjectif mais de l'aborder à la manière d'une production " équipée », pouvant atteindre un degré de scientificité se matérialisant dans des pratiques concrètes. Dès lors, les mobilisations collectives ne sont donc pas simplement l'expression d'une revendication, elles sont aussi potentiellement une objectivité en devenir [Tournay, 2007b].

Les travaux qui suivent sont réunis par un même axe thématique : les revendications sociales liées au domaine de l'innovation médicale. La forte instabilité des savoirs en question et la grande sensibilité sociale de ces sujets constituent une porte d'entrée pertinente pour saisir la manière dont des groupes sociaux vont convaincre, c'està-dire produire une évidence collective partagée, directement intégrable dans les politiques publiques sous la forme de préceptes ou d'opérateurs concrets. En partant des différentes formes d'activisme médical, de leurs répertoires argumentatifs et pratiques, on peut alors décrire les linéaments des agencements sociotechniques qui s'affirment progressivement, qu'ils s'agissent d'une pratique en voie de légitimation (la contraception : article Sanseigne), de la médicalisation d'un domaine disciplinaire (les soins palliatifs : article Castra), de l'objectivation d'une expertise associative (les centres de santé associatifs : article Warin), de la consolidation d'une catégorie nosologique (l'autisme : article Chamak ou le SIDA : article Musso) ou d'une production thérapeutique (médicaments pour maladies orphelines : article Novas). Des notes de recherche en cours témoignent de l'importance des espaces locaux de monstration dans la stabilisation de collectifs (les sourds : article Dalle-Nazébi et Lachance et les 
surdoués : article Lignier) et la standardisation de procédures (observance aux multithérapies : article Gilbert).

Au double présupposé précédemment énoncé, s'ajoute un parti pris phénoménologique. Les instruments de la monstration ne sont pas uniquement des matériaux sensoriels apparaissant au sujet comme des formes visuelles ou des qualités sensibles dépourvues de significations en soi. Les scènes de monstration sont des actes de faire percevoir ; ce sont des médiations ayant une effectivité pratique car elles détiennent intrinsèquement une valeur fonctionnelle déterminée et une interprétation propre liées aux formes, aux propriétés physiques des objets et aux évènements immédiatement perçus [Gibson, 1979, Quéré, 1999]. Par exemple, la matérialité et la scénographie des opérations électorales sont, dans leur exécution même, un acte de persuasion visant à témoigner de la qualité de la démocratie représentative [Déloye et Ihl, 2008]. Ce constat peut être étendu aux dispositifs participatifs dont l'organisation compose avant tout des tentatives de figurer un public plus ou moins étendu, fragile, éphémère ou consolidé [Brugidou, 2008]. La perception du monde extérieur est donc indissociable d'une perception de significations et de déterminations plus ou moins équivoques [Wittgenstein, 1999]. Ainsi, l'inscription de catégories sous forme de listes, de tableaux tend à dépersonnaliser la langue parlée et à faire adopter une apparence de neutralité : enregistrement des naissances, des décès, d'une nosographie nouvelle (Brigitte Chamak) ; autant de raisons graphiques qui fournissent une apparente autonomie des énoncés par rapport à leurs conditions historiques de production [Goody, 1979] et qui ouvrent la voie à une sociologie historique de l'argument statistique et de la quantification [Desrosières, 2008]. Catégoriser c'est également mettre de la similitude entre des choses auparavant différentes comme en témoigne la grande variété culturelle des classifications nosographiques, en particulier dans le domaine de la maladie mentale (Brigitte Chamak). Lorsqu'elle fait preuve, la monstration peut donc être considérée comme un objet frontière [Star et Griesemer, 1989], véritable arène interstitielle [Shinn et Ragouet, 2005] susceptible de mettre en place des mécanismes de coopération et d'établir des instruments d'équivalence entre des acteurs issus de mondes sociaux ou d'univers de sens différents. On le voit très nettement dans l'analyse de Philippe Warin où les centres de santé associatifs doivent établir des bonnes pratiques de qualité devant satisfaire des acteurs et des exigences hétérogènes. En suivant la production d'une conviction partagée et durable dans les milieux associatifs et des formes émergentes d'expertise [Jacob et Genard, 2004]", ce type d'analyse est ainsi exemplaire pour faire rencontrer la sociologie des mobilisations collectives à celle de l'action publique [Hamel, Maheu et Vaillancourt, 20007 ; Tournay, 2007b ; Cantelli, 2007]. En bref, derrière les travaux rassemblés, ce numéro de Quaderni pose en principe le postulat suivant : Revendiquer, protester, instituer : c'est avant tout faire percevoir. Au lecteur de juger s'il doit céder à la monstration et s'y laisser envoûter... 
$R \cdot E \cdot F \cdot E \cdot R \cdot E \cdot N \cdot C \cdot E \cdot S$

M. BRUGIDOU, L'opinion et ses publics, Paris, Presses de Sciences Po, 2008.

S. CADIOU et S. DECHEZELLES, « La problématique de l'émergence pour l'étude des mobilisations collectives : Pistes et repères », in Passer à l'action : Les mobilisations émergentes. Paris, L'Harmattan, Logiques Politiques, 2007, pp. 11-48. F. CANTELLI, L'État à tâtons. Pragmatique de l'action publique face au SIDA, Berne/Bruxelles, 2007.

Y. DÉLOYE et O. IHL, L'acte de vote, Paris, Sciences Po, 2008.

A. DESROSIÈRES, L'Argument statistique; Vol.1 et 2, Pour une sociologie historique de la quantification et Gouverner par les nombres. Paris, Presses de l'école des mines, 2008.

J. DEWEY, Logique; la théorie de l'enquête. Paris, Presses universitaires de France, 1967.

N. DODIER et J. BARBOT, «Autonomy and Objectivity as Political Operators in the Medical World: Twenty Years of Public Controversy about AIDS Treatments in France "), in Science in Context, 21, 2008, pp. 403-434.

J. DUMIT, Picturing Personhood: Brain Scans and Biomedical Identity, Princeton University Press, 2003.

O. FILLIEULE, Sociologie de la protestation - Les formes de l'action collective dans la France contemporaine, L'Harmattan, Paris, 1993.

N. FRIEDMAN, The social nature of psychological research : the psychological experiment as a social interaction, Basic Books, New York, 1967.

J.-J. GIBSON, The ecological approach to visual perception, Boston, Houghton Mifflin, 1979.
O. GIRAUD et P. WARIN, « Les politiques publiques: Une pragmatique de la démocratie », in Politiques publiques et démocratie, (dir. O. Giraud et P. Warin), La Découverte/PACTE, pp. 7-31.

E. GOFFMAN, Façons de parler, Paris, Éditions de Minuit, 1987.

J.-R. GOODY, La raison graphique : la domestication de la pensée sauvage, Paris, Éditions de Minuit, 1979.

P. HAMEL, L. MAHEU et J.-G. VAILLANCOURT, « Repenser les défis institutionnels de l'action collective », in Politique et Sociétés, vol. 19, n¹, 2000, pp. 3-25.

S. JACOB et J.-L. GENARD (eds), Expertise et action publique, Bruxelles, Éditions de l'ULB, Collection sociologie politique, 2004,

P. LASCOUMES et P. Le GALÈS. Gouverner par les instruments, Paris, Presses de la fondation nationale des sciences politiques, 2004.

C.W. MILLS, «Situated Actions and Vocabularies of Motive », in American Sociological Review, 5(6), 1940, pp. 904-913.

V.-K. NGUYEN, «Antiretroviral Globalism, Biopolitics, and Therapeutic Citizenship », in Global Assemblages - Technology, Politics, and Ethics as Anthropological Problems, Blackwell Publishing, 2005, pp. 124-144.

R. PRENTICE, "The Anatomy of Surgical Simulation », in Social Studies of Science, vol. $35, n^{\circ} 6,2005$, pp. 837-866.

L. QUÉRÉ, "Action située et perception du sens », M. de Fornel et L. Quéré (éds.), in $L a$ logique des situations, EHESS, Paris, 1999, pp. 301-338.

L. SFEZ, « La politique symbolique des Quaderni », in Quaderni, n³8, printemps 1999, pp. 117-124. 
T. SHINN et P. RAGOUET, Controverses sur la science, Paris, Raisons d'agir, 2005.

J. SIMÉANT, «Friches, hybrides et contrebandes : sur la circulation et la puissance militantes des discours savants ", in Discours savants, discours militants : mélange des genres, L'Harmattan, 2002, pp. 17-53.

A. SCHUTZ, Reflections on the problem of relevance. New Haven, Yale University Press, 1970.

S.-L. STAR et J. GRIESEMER, « Institutional ecology, translation and boundary objects : amateurs and professionals in Berkeley's Museum of Vertebrate Zoology 1907-1939 », in Social Studies of Science, 19, 1989, pp. 387-420.

G. TARDE, Fragment d'histoire future, Chicoutimi, J.-M. Tremblay (pub.reprod.), 2003. J. TAYLOR, The Public Life of the Fetal Sonogram: Technology, Consumption, and the Politics of Reproduction, Rutgers University Press, 2008.

V. TOURNAY, « Le concept de police médicale. D'une revendication militante à la production d'une objectivité administrative", in Politix, n77, vol. 19, 2007a, pp. 175-201.

V. TOURNAY, « Comment inscrire durablement les revendications militantes, les pratiques sociales et les récits historiques?», V. Tournay (dir.), in La Gouvernance des Innovations Médicales, Paris, PUF, 2007b.

D. TROM, « De la réfutation de l'effet NIMBY considérée comme une pratique militante-Note pour une approche pragmatique de l'activité revendicative », in RFSP, Vol. 49, No. 1, 1999, pp. 31-50.

L. WITTGENSTEIN, Remarques sur la philosophie de la psychologie, Mauvezin, T.E.R., 2 tomes, 1989 et 1994.
N

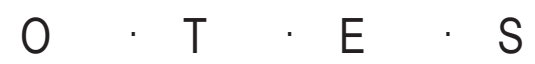

1. La publication de ce numéro a bénéficié de l'aide de l'Agence Nationale de la Recherche et de l'Agence de la Biomédecine (Projet JC08_318187. THERACELS). Elle s'inscrit dans le Pôle des Approches Pragmatiques en Science-Politique (PAPS) de l'UMR PACTE.

2. Le symbolique désigne un mode d'intellection par lequel nous nous lions aux phénomènes pour les subsumer sous des catégories, les lier entre eux et ainsi les comprendre, c'est-à-dire les intégrer dans un tableau de faits. [Sfez, p. 119]. C'est donc un terme éminemment politique.

3. Pour l'auteur, "accomplir une action, c'est introduire dans un milieu quelque chose qui va circuler $»$.

4 Les deux auteurs établissent la généalogie de deux opérateurs politiques: le concept d'autonomie du patient et l'objectivité de la prise de décision médicale. Ils décrivent les tensions autour de ces notions dans l'histoire des controverses autour du SIDA.

5. La construction des représentations médicales du corps et leurs caractérisations impliquent une articulation spécifique entre les corps observés, les instruments déployés et l'individu observant.

6. Pour une discussion sur la construction de l'action publique et les expertises associées.

7. Pour toute une réflexion sur l'institutionnalisation des mouvements sociaux et sur les rapports entre les théories de l'institutionnalisation et celles de l'action collective. 
